\title{
Desarrollo humano, democracia, ciudadanía y diversidad y justicia social
}

En los actuales contextos de las sociedades democráticas, el derecho consignado de ciudadanía redunda en un trasfondo de conceptos y preceptos que van desde la parte fundamental consignada en la Constitución Política de una nación, los derechos humanos, pasando por el marco legislativo que incluye a la ética en el ejercicio de los derechos ciudadanos.

Se puede tener como una de las aristas de tal problemática, el asumir con tácita hidalguía que se tiene una precariedad estructural en el ejercicio de la ciudadanía, la cual una parte deriva de las instituciones más representativas del Estado, al asumir desde la educación hasta las garantías jurídicas. En relación con las minorías étnicas y las comunidades rurales, se hace patente un débil a nulo compromiso político por parte del Estado hacia aquellos ciudadanos, muchos de los cuales por diversos motivos están privados de representatividad ante las instancias correspondientes. De lo anterior se desprende que, si la institucionalidad no es patente o presenta deficiencias de facto, los derechos solo son representaciones discursivas, mas no efectivas. Dicho predicamento descarna una completa ausencia de Estado, diagnosticándose una metástasis cancerbera en las instituciones estatales que bloquea el acceso y el ejercicio pleno de los derechos.

Si bien el ideal del ciudadano con el ejercicio pleno de todos sus derechos en una sociedad libre y justa, basada en preceptos de democracia e igualdad es la visión prima de las leyes al respecto, cabe resaltar que no siempre se puede vislumbrar tal requisito de anhelo compartido. La desigualdad del desarrollo en los países americanos es una tara onerosa y que, a pesar de haber batallado por la anhelada libertad e independencia de los pueblos, aún la tarea no está concluida. Tal como lo mencionara el poeta universal César Vallejo: "Hay hermanos muchísimo que hacer"; la frase impuesta es hoy asumida como una insigne consigna, por la cual las vertientes del pensamiento humano se enfocan en el estudio, análisis y divulgación de los menesteres y predicamentos, así como los logros y conquistas logradas en pos de un ciudadano, del ser humano pleno en el respeto irrestricto de sus derechos, con la capacidad de ensalzar la añorada justicia social que es el derrotero por el cual frente a la gran diversidad de nuestros pueblos, diversidad compleja pero que en problemáticas sabe tan crónica.

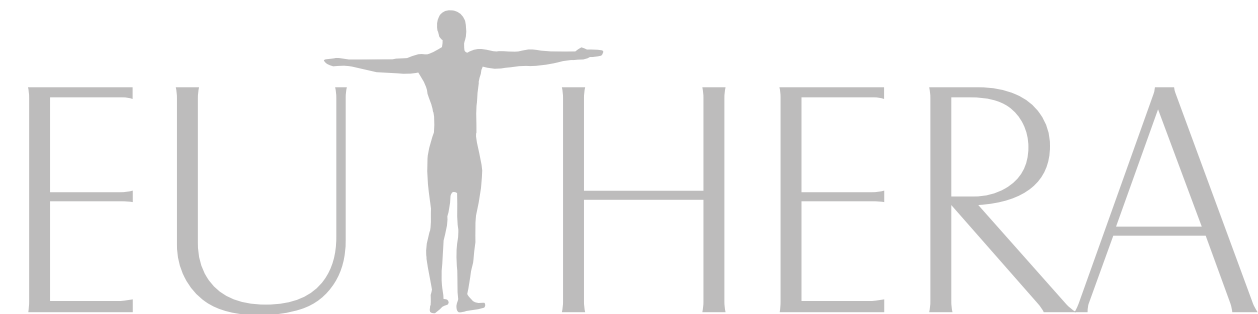


Es notable el conceso que la actual coyuntura brinda para la apertura de análisis y debates sobre la ciudadanía, derechos humanos, respeto a las diversidades y justicia social con relación a su trascendencia del desarrollo de los pueblos y la lucha contra la desigualdad, pobreza y discriminación, lastres que aquejan los pueblos cada vez con mayor repercusión. Por ello, plantear mecanismos y alternativas que se avoquen en plantear soluciones viables para facilitar canales hacia el ideal de ciudadanía, brindaría un vínculo efectivo para constituir vías de acceso para alcanzar el goce concreto de todos los derechos. Para ello, es imprescindible que el Estado y sus instituciones emblemáticas tiendan a catalizar sus esfuerzos por la mejora sustantiva en educación, salud y respeto al medio ambiente y manejo sostenido de recursos; por ello, los estándares de operatividad, eficiencia, eficacia en la gestión de dichos estamentos debe ser tomado muy en cuenta, a fin de garantizar la aplicabilidad de los derechos ciudadanos y deje de ser considerada como una tarea pendiente en las agendas de políticas de desarrollo estatal.

Hay que tener muy presente que en la praxis social es menester un trabajo compartido para constituir la democracia y la ciudadanía. El desarrollo de un Estado social de derecho no es producto de la voluntad presidencial, sino de la articulación virtuosa del Estado y la sociedad. Para entender esto es necesario tener claro el concepto de ciudadanía. Los invito a leer esta edición con los ojos de nuestros presentes y futuros retos.

En la primera sección del presente volumen Desarrollo Humano, Democracia y Ciudadanía contamos con nueve artículos que dan cuenta, principalmente, de temáticas relacionadas con estudios educativos, pensamiento crítico, prácticas inclusivas, competencias digitales en educación y demás. El primero de estos artículos se titula "Apreciación del docente para contribuir al desarrollo del pensamiento crítico”, escrito por María Patricia Gómez Gómez y Sandra Milena Botero Bedoya, tiene como propósito presentar algunos métodos de enseñanza y estrategias educativas que pueden contribuir al desarrollo del pensamiento crítico. Estas autoras encontraron que, por medio del desarrollo del pensamiento crítico en el aula a través de estrategias de aprendizaje, los profesores pueden contribuir de manera significativa a la capacidad de organizar, sistematizar las ideas y sintetizar criterios elementales, de manera razonable, reflexiva y coherente en los estudiantes.

El segundo artículo del presente volumen, "Estrategias didácticas en el desarrollo del pensamiento crítico en estudiantes de educación básica” de los autores Luis Alberto Núñez Lira, Dally Mayer Gallardo Lucas, Alicia Agromelis Aliaga Pacore y Jorge Rafael Díaz Dumont. El objetivo del presente documento es evaluar, por medio de un análisis tanto cuantitativo como cualitativo, las estrategias didácticas usadas en las sesiones de aprendizaje del área de comunicación para desarrollar el pensamiento crítico en estudiantes de educación secundaria de instituciones educativas de la jurisdicción de Ocros, a partir de estrategias para desarrollar la creatividad y pensamiento de los estudiantes. 
El tercer artículo se titula "Estrategias didácticas para desarrollar prácticas inclusivas en docentes de educación básica”, escrito por Jessica Paola Palacios Garay, Violeta Cadenillas Albornoz, Patricia Gladys Chávez Ortiz, Ruth Alina Flórez Barrios y Karol Moira Abad Escalante. En este artículo, las autoras establecen estrategias didácticas en el desarrollo de prácticas inclusivas en docentes de educación básica regular por medio de un estudio positivista, de enfoque cuantitativo, tipo sustantivo, diseño no experimental y correlacional causal. Como resultado afirman que se evidenciaron bajos niveles del uso de estrategias didácticas para desarrollar prácticas inclusivas en docentes de educación básica.

El cuarto artículo de la presente sección "Desarrollos de competencias digitales en docentes universitarios a través de entornos virtuales: experiencias de docentes universitarios en Lima” escrito por Carlos Miguel Laurente Cárdenas, Raúl Alberto Rengifo Lozano, Nicanor Segismundo Amad Vega y Lida Neyra Huamani. Los autores proponen allí el uso de entornos virtuales reflexiva y críticamente por parte de los docentes, en el que el desarrollo de competencias digitales se configure como transversal en la interacción con espacios virtuales durante su praxis profesional y aprendizaje continuo.

Seguidamente está el artículo “Sobre la gobernanza digital, política digital y educación” de los autores Luis Alex Valenzuela Fernández, Zoila Mercedes Collantes Inga y Eduardo Ernesto Durand, quienes hacen una revisión acerca de los fundamentos orientados a la política digital y sus mecanismos de gobernanza desde una nueva perspectiva, tomando en cuenta las vertientes que se vienen desarrollando, los argumentos a favor y en contra con los que se han topado en su aplicación. Por otro lado, se examina explícitamente los aspectos relacionados al desarrollo de las políticas digitales aplicadas al campo de la educación, al abarcar diversos nexos tales como lo ideológico, administrativo y aplicativos de este novedoso asunto, analizando la problemática y soluciones desde la perspectiva de la gobernanza digital. Finalmente, se discuten las oportunidades y dificultades del desarrollo de los sistemas de gobernanza multinivel, con base en la aplicación de infraestructuras digitales y la consolidación de ecosistemas tecnológicos urbanos.

El sexto artículo, escrito por Jorge Ernesto Torres Obleas y Daniela Medina Colorado, titulado "Eficacia de los objetos virtuales para el aprendizaje en el uso de estrategias de lectura de estudiantes de distritos del Perú con restricciones en conectividad y equipamiento. Caso Villa Rica” analizan cómo el uso de tecnologías de información y comunicación en Perú han mejorado la educación y, por ende, el desarrollo humano. No obstante, afirman que existen poblaciones que carecen de conexiones a internet, como es el caso de Villa Rica; en ese sentido proponen, mediante una propuesta cuasi experimental, determinar el efecto del nivel de interactividad de los objetos virtuales para el aprendizaje, OVA, en la adquisición de estrategias de lectura relevante para el diseño de OVA, al estar la interactividad condicionada por la conectividad. 
Después está el artículo "Intervención educativa basada en la gamificación: experiencias en el contexto universitario” de Mitchell Alberto Alarcón Díaz, Henry Hugo Alarcón Díaz, Liset Sulay Rodríguez Baca y Noel Alcas Zapata, cuyo objetivo fue identificar la existencia de diferencias estadísticas significativas en la aplicación de la gamificación como estrategia didáctica en estudiantes universitarios de una universidad privada de Lima. Los autores afirman que existen diferencias estadísticas significativas entre el grupo experimental y el grupo control y los alumnos que experimentaron a través de la gamificación muestran mejores resultados.

Finalmente, está el artículo "Participación ciudadana como estrategia de diagnóstico para la toma de decisiones para reducir la desnutrición crónica en un distrito de Lima” de Jacinto Joaquín Vértiz Osores, María Fanny Aparicio Fernández, Manuel Felipe Guevara Duarez, Jorge Luis López Bulnes, Segundo Pérez Saavedra y Javier Arturo Gamboa Cruzado. En este, los autores tienen como objetivo conocer la pertinencia de la participación ciudadana como estrategia en la toma de decisiones en la reducción de la desnutrición crónica por medio de un estudio fenomenológico basado en entrevistas. Los autores argumentan que es necesario visualizar la participación ciudadana como estrategia en la toma de decisiones por parte del Estado, directivos o funcionarios públicos, dado que es un medio efectivo de la comunicación entre estos.

En la segunda sección del presente volumen, Diversidad y Justicia Social, se presentan siete artículos. El primero se titula "Factores de delincuencia juvenil en el Perú desde el enfoque preventivo" escrito por Freddy Ccopa Quispe, Doris Fuster Guillén, Katelinen Rivera Paipay, Yadiri Pejerrey Rivas y Miguel Tupac Yupanqui Bustamante. En este escrito, los autores identifican los factores predominantes que provocan que la juventud cometa actos delincuenciales en el distrito de Villa María del Triunfo en Perú.

El segundo artículo "Acción colectiva, antagonismos y resistencia comunitaria en el conflicto socioterritorial de Freirina” de Cory Duarte Hidalgo, Carolina López Rojas, Paloma Ochoa García y Paula Salazar Enríquez, analiza la acción colectiva desarrollada en el marco del conflicto socio-territorial en la localidad de Freirina, Atacama, Chile. La Asamblea Freirina articuló una resistencia comunitaria como respuesta al funcionamiento de una planta faenadora y procesadora de cerdos, lo que implicaba una serie de consecuencias para el territorio y la comunidad. Las autoras observaron el despliegue de distintos repertorios de acción, la existencia de relaciones antagónicas suscitadas en el conflicto, la importancia los espacios y territorios locales y la resistencia comunitaria en la lucha por la dignidad.

Después está el artículo "Nuevas ruralidades como reconfiguración del territorio en transformación: imaginarios sociales corregimentales a la luz de organizaciones socioculturales", autoría de Natalia Andrea Salinas Arango y Mateo Sanmartín Gaviria. En este escrito los autores identifican, por medio de un estudio cualitativo, los imaginarios sociales que se tienen sobre 
las transformaciones de lo rural a lo urbano y su incidencia en el desarrollo local desde la perspectiva de cuatro organizaciones socioculturales.

El cuarto artículo de la presente sección "Importancia de la diferenciación del enfoque diferencial étnico en el proceso de reparación de Bojayá-Chocó” de la autora Wanny Elizabeth Hinestroza Ramírez. Aquí, argumenta que la aplicación del enfoque diferencial étnico en la reparación a víctimas negras tiene una trascendencia reivindicatoria de los derechos humanos como grupo, al constituirse como un instrumento de reconocimiento de sus particularidades culturales. Este artículo tiene por objetivo hacer una reflexión crítica a la aplicación de los principios y directrices que orientan la reparación en el derecho internacional, estudiando de manera particular el caso de Bojayá.

Después está el artículo titulado "Emociones, conflicto y educación: bases para pensar la educación emocional para la paz” de Gina Paola Alzate Henao, Mónica María Bedoya Rojas, Aura María Fajardo Sandoval, Ángela del Pilar Hoyos Mejía y Esteban Ocampo Flórez, cuyo objetivo es analizar, por medio de una exhaustiva revisión bibliográfica, tres categorías conceptuales presentes en la investigación "Educación emocional para la paz en ambientes escolares, una aproximación desde las percepciones de los actores”, a saber: emociones, conflicto y educación. Los autores concluyen que una educación que responda a los desafíos de la época asume el desarrollo socioafectivo, una concepción positiva de conflicto y entornos de aprendizaje significativo y colaborativo, como elementos esenciales para la construcción de sociedades pacíficas, solidarias y equitativas.

El quinto artículo de la presente sección, escrito por Diana Marcela Alzate Torres, se titula "De la tensión en el ejercicio de los roles de género a la violencia conyugal como pauta relacional”. En este artículo se presentan los resultados de una investigación sobre violencia conyugal, por medio de un enfoque cualitativo desde la perspectiva hermenéutica, realizada con parejas de la ciudad de Manizales, cuyo propósito fue analizar las conexiones entre tensiones generadas en el ejercicio de los roles de género y la emergencia de la violencia en la relación de pareja.

El último artículo de la presente sección "Parkour y skateboard: intersubjetividad en las calles de la ciudad de Bogotá", escrito por Jhon Carlos Cortés Murillo, tiene como propósito interpretar los sentidos y significados que subyacen en las relaciones que los practicantes de parkour y skateboard tejen dentro de su práctica y con los demás habitantes de la ciudad. Los resultados indican que las relaciones intersubjetivas en la práctica se caracterizan por su horizontalidad, en ellas los saberes y experiencias valen por su carácter formativo y amistoso y no por el prestigio y jerarquía que otorgan; cuando se trata de pares, la interrelación de los sujetos está circunscrita a la solidaridad, la responsabilidad, la motivación y cuidado del otro. 
En la última sección, Enfoques en Trabajo Social: Investigación y Práctica, profesional se presenta un artículo denominado "Prácticas de resistencia y trabajo social comunitario: forcejeos y tensiones ante las lógicas de dominación del modelo colonial y capitalista” de Carmen Gloria Jarpa Arriagada. Este artículo de reflexión crítica deriva del trabajo desarrollado por la autora en el Centro de Intervención e Investigación Social (CIISETS) en Chillán, Chile. Específicamente, se discute la experiencia derivada del trabajo comunitario de inserción territorial y sus tensiones actuales. El debate teórico transita desde conceptos de dominación y resistencia, hasta el análisis de lo decolonial y el Trabajo Social-Otro. La propuesta de práctica de resistencia al modelo neoliberal la construyó desde un trabajo social ético-político emancipatorio que logra sinergia entre la recuperación de los saberes populares y la investigación activista como compromiso para la producción de conocimiento.

Ronald M. Hernández

Universidad San Ignacio de Loyola, Lima, Perú

rhernandezv@usil.edu.pe

https: / / orcid.org/0000-0003-1263-2454

Yolvi Ocaña-Fernández

Universidad Privada San Juan Bautista, Lima, Perú

yolvi.ocana@upsjb.edu.pe

https: / / orcid.org/0000-0002-2566-6875 